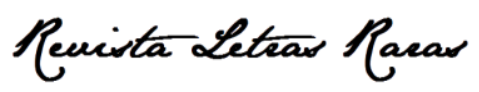

ISSN: 2317-2347 - v. 9, Dossier spécial (2020)

Todo o conteúdo da RLR está licenciado sob Creative Commons Atribuição 4.0 Internacional

\title{
Le travail collaboratif et bénévole en réseau : enjeux actuels de la
}

\section{Fédération Brésilienne des Professeurs de Français / \\ O trabalho colaborativo e voluntário em rede: embates atuais da}

Federação Brasileira dos Professores de Francês

\section{Denise Gisele de Britto Damasco *}

Licenciée en Lettres (1986), ayant un Master Recherche (2008) et Doctorat (2014) en Education à l'Université de Brasilia. Enseignante de français langue étrangère au Secrétariat d'Etat d'Education du DF de 1989 à 2015. Actuellement, suit des études postdoctorales à la PUC SP et préside la Fédération brésilienne des professeurs de français (2017-2020 et 2020/2022).

iD http://orcid.org/0000-0002-0250-0776

\section{Laurizete Ferragut Passos **}

Licenciée en Pédagogie. A obtenu un Master Recherche à l'Université Unicamp et un Doctorat en Sciences de l'Éducation à l'Université de São Paulo. Coordonne le Programme d'Études de Pós-Graduação en Education Master Professionnel Formation des Formateurs de la PUCSP. Maître de Conférences du Programme d'Études de Pos-Graduação en Psychologie de l'Education de la PUCSP.

iD https://orcid.org/0000-0001-7702-0825

Reçu le : 11 oct. 2020. Approuvé le: 24 oct. 2020.

\section{Comment citer cet article:}

DAMASCO, Denise Gisele de Britto; PASSOS, Laurizete Ferragut. Le travail collaboratif et bénévole en réseau : enjeux actuels de la Fédération Brésilienne des Professeurs de Français. Revista Letras Raras. Campina Grande, v. 9, p. 179-192, oct. 2020.

\section{RÉSUMÉ}

Cet article présente le travail collaboratif et bénévole en réseau qui se dévoile à travers les associations de professeurs de français, qui par la suite forment une fédération nationale. La Fédération brésilienne des professeurs de français réunit 23 associations d'enseignants, étudiants de licence de Lettres, de Traduction, entre autres et des professionnels qui

*

$\triangle$ denise.damasco@gmail.com

$\triangle$ laurizetefer@gmail.com 


\section{Reuista Letear Pacar}

ISSN: 2317-2347 - v. 9, Dossier spécial (2020)

Todo o conteúdo da RLR está licenciado sob Creative Commons Atribuição 4.0 Internacional

promeuvent le français au Brésil dans le but de cibler des actions communes et de renforcer l'enseignement de la langue française à partir des liens nationaux et internationaux. Pour comprendre ce travail collaboratif et bénévole en réseau, nous aimerions d'abord présenter quelques éléments de l'histoire de la FBPF, puis les défis des associations solides dans le pays et des associations débutantes, y compris celles qui recommencent leurs activités associatives. Nous souhaiterions expliciter le rôle des associations des professeurs de français dans la formation continue des enseignants et dans l'insertion professionnelle des enseignants novices. Pour cela, nous rendrons compte de quelques expériences dans les sphères associative, fédérative et de la coopération éducative francophone en partenariat pour faire avancer et renforcer la profession enseignante en Français Langue Étrangère (FLE) dans le pays.

MOTS-CLÉS : Association de professeurs ; Enseignement de français langue étrangère ; Travail collaboratif ; formation continue ; insertion professionnelle

\section{RESUMO}

Este artigo reflete sobre o trabalho colaborativo e voluntário que acontece em rede, ou seja, aquele realizado por meio das associações de professores de francês que, por sua vez, formam uma federação nacional. A Federação brasileira dos professores de francês (FBPF) é associada à Federação internacional dos professores de francês, cujos objetivos comuns são o fortalecimento da carreira e formação docente em francês. A FBPF reúne 23 associações de docentes, estudantes de licenciatura em Letras, em Tradução entre outros cursos, e profissionais atrelados à promoção do francês no Brasil com metas de focar ações comuns para o fortalecimento do ensino do francês a partir de laços nacionais e internacionais. Para compreender o trabalho colaborativo e voluntário em rede, gostaríamos de, primeiramente, apresentar alguns elementos da história da FBPF, em seguida, os desafios das associações sólidas no país, as associações que iniciam suas atividades, dentre as quais aquelas que retomam suas atividades associativas. Finalmente, explicitamos o papel das associações dos professores de francês e da FBPF na formação continuada e na inserção profissional de professores iniciantes. Para tal, apresentamos algumas experiências nas esferas associativa e federativa, bem como de parceria em cooperação educativa francófona no intuito de fazer avançar e reforçar a profissão docente em francês no país.

PALAVRAS-CHAVE: Associação de Professores; Docência em francês língua estrangeira; Trabalho colaborativo; Formação continuada; inserção profissional

\section{Introduction}

Cet article présente une réflexion sur le travail collaboratif et bénévole en réseau réalisé par la Fédération brésilienne des professeurs de français (FBPF), qui réunit 23 associations d'enseignants, étudiants en licence de Lettres, de Traduction, entre autres ainsi que des professionnels liés à la promotion du français au Brésil dans le but de cibler des actions communes et de renforcer l'enseignement de la langue française à partir des liens nationaux et internationaux. Le réseau professionnel créé par l'Institut Français, comme politique publique pour l'éducation en français et pour le français a invité la FBPF pour devenir son partenaire institutionnel sur sa plateforme au Brésil, étant donné son rôle majeur dans la promotion du français et dans la formation des enseignants de FLE au Brésil à partir du réseau fédératif brésilien et international ${ }^{1}$.

\footnotetext{
1 La thématique de cet article a été présenté le 15 juillet 2020 lors du lancement du partenariat institutionnel entre la FBPF et l'Institut Français - Ambassade de France au Brésil.
} 


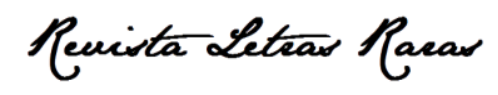

ISSN: 2317-2347 - v. 9, Dossier spécial (2020)

Todo o conteúdo da RLR está licenciado sob Creative Commons Atribuição 4.0 Internacional

Nous pourrions donc esquisser un effet de cascade qui se reproduit dans le réseau mondial d'enseignants de français, autrement dit : Les associations de professeurs brésiliennes sont associées à la FBPF qui est membre associée de la Fédération internationale des professeurs de français (FIPF). Cette thématique englobe alors le terme « adhérer », qui signifie devenir membre, être associé, fédéré. C'est un choix, une décision personnelle de participer à une association. Pour que l'association adhère à une fédération, il faut qu'un groupe dise oui au projet fédérateur qui rassemblera des objectifs communs. Une association de professeur de français n'est pas une entreprise, ni un groupe de recherches, ni une école de langues, mais un groupe de professionnels qui a décidé de se réunir pour avancer ensemble à travers le bénévolat et la convivialité. Une association est une entité juridicofiscale dont les membres de son bureau sont responsables des actions votées pendant l'Assemblée Générale, une vraie action vers la citoyenneté : collaborer de façon bénévole.

Pour saisir ce travail collaboratif2 et bénévole en réseau, nous aimerions d'abord définir ce que nous comprenons par travail collaboratif et l'importance des échanges et des espaces de partages dans le développement professionnel. Ensuite, nous souhaiterions expliciter quelques éléments de l'histoire de la FBPF, suivis des défis des associations solides dans le pays et des associations débutantes, y compris celles qui recommencent leurs activités associatives. Nous expliciterons enfin le rôle des associations des professeurs de français dans la formation continue des enseignants e et dans l'insertion professionnelle des enseignants novices. Pour cela, nous envisageons de présenter quelques expériences dans les sphères associative, fédérative et de la coopération éducative francophone en partenariat pour faire avancer et renforcer la profession enseignante en FLE dans le pays.

\section{Le travail collaboratif : qu'est-ce que cela veut dire ?}

Tout d'abord, nous aimerions affirmer qu'il existe une théorie assez solide sur ce qu'est le travail collaboratif, non nécessairement un travail bénévole. Collaborer indique qu'un travail est mené avec une ou plusieurs personnes dans la réalisation d'une œuvre commune. Le travail bénévole

2 La théorie concernant le travail collaboratif et groupes collaboratifs a été étudiée lors du stage postdoctoral en éducation en 2020 mené à la PUC de São Paulo. 


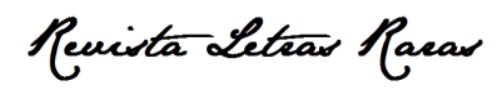

ISSN: 2317-2347 - v. 9, Dossier spécial (2020)

Todo o conteúdo da RLR está licenciado sob Creative Commons Atribuição 4.0 Internacional

consiste à faire quelque chose sans être obligé, sans en tirer profit. II faut souligner qu'en langue française il existe une différence entre bénévolat et volontariat. Le travail bénévole indique qu'il a été réalisé avec $100 \%$ de gratuité. Le travail volontaire indique que le participant a dû recevoir une aide pour le réaliser, une rémunération pas très importante, plutôt symbolique, juste pour rembourser des dépenses minimales. Cette distinction n'existe pas en langue portugaise.

Bois et Portelance (2014) ont présenté leurs études sur la collaboration en milieu d'accueil des enseignants débutants. Les auteures reconnaissent qu'il existe des difficultés au début de la carrière qui peuvent mener à l'abandon de la profession. Selon leurs interprétations des résultats de leur recherche, la qualité des interactions est au cœur de la construction identitaire professionnelle. Cet article ne prétend pas approfondir cette thématique liée à la collaboration dans le milieu scolaire mais en milieu associatif et fédératif.

Ce n'est pas parce que nous sommes ensemble que nous avons les mêmes objectifs. II faut penser d'une façon stratégique pour collaborer (FULLAN ; HARGREAVES, 2000), où ils travaillent, leur groupe professionnel et de quoi ils ont besoin. Discuter sur les problématiques des enseignants, échanger des pratiques, se réunir une fois par an ne veut pas dire que nous collaborons et que nous avons un projet commun (LITTLE, 1990 apud FULLAN ; HARGREAVES, 2000). Un ensemble d'actions isolées ne signifie pas que nous sommes en collaboration. L'isolement bloque le développement professionnel et empêche les échanges et le partage (FULLAN ; HARGREAVES, 2000).

Le travail collaboratif signifie prendre des décisions ensemble ayant la participation de toutes et tous, de façon démocratique, penser à une action commune. Participer à un groupe collaboratif, à un groupe de recherches, à une communauté de pratiques ou à une communauté d'apprentissage entamer des actions telles que se poser des questions, dialoguer, se former ensemble, échanger et partager. Occuper un espace et lui rendre du sens pour transformer la situation, pour avancer (PASSOS, 2016). En groupe, nous avons plusieurs regards et opinions. Nous pouvons devenir moins isolés(es) et valoriser l'écoute de l'autre.

Calvo (2018) affirme que les enseignants ne partagent pas les mêmes moments de leur carrière enseignante ni les mêmes moments de formation. Écouter l'autre, ses expériences, ses défis, sa pratique peuvent nous faire comprendre nos parcours et nos propres difficultés (PASSOS, 2016). II y a un apprentissage professionnel collaboratif et des échanges entre les pairs. Cela entraîne un 


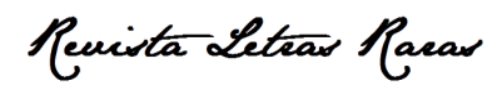

ISSN: 2317-2347 - v. 9, Dossier spécial (2020)

Todo o conteúdo da RLR está licenciado sob Creative Commons Atribuição 4.0 Internacional

risque, selon Fullan et Hargreaves (2000), puisqu'il nous faut un cadre où il y a la confiance, la valorisation de l'autre ainsi que la reconnaissance du partage des savoirs, la quête de conseils et une entraide. II y a donc un impact sur nos incertitudes dans notre domaine professionnel - notre havre de paix.

Être en groupe ne veut pas dire que tout se passera bien, ce n'est pas un passeport pour la réussite sachant que les enseignants peuvent devenir de meilleurs professionnels ou moins bons. Selon Fullan et Hargreaves (2000), il y a des problèmes qui surgissent quand nous sommes en groupes. II se peut que l'effet "comptoir" soit présent, un rapport entre des clients et des commerçants. Plusieurs sous-groupes qui cherchent des avantages peuvent apparaître ou la perpétuité des groupes qui empêchent de nouveaux arrivants. II suffit de privilégier les mêmes connexions pour que le travail collaboratif soit confortable, sans défis, sans échanges et sans conflits : collaborer avec ceux et celles que j'aime ou avec qui je veux interagir ne nous semble pas être la recette de la réussite en termes de collaboration.

Pour le développement professionnel, la chercheuse Calvo (2018) affirme qu'il faut du temps ; des ressources pédagogiques, des conseils, un appui, une aide, un parrainage, un coup de main. II nous faut également des opportunités, du suivi, de l'évaluation et de l'autoévaluation et des motivations. Commencer par des actions bénévoles peut déclencher ce processus.

\section{3. Être en réseau pour faciliter le transfert de savoir : le cas de la FBPF}

Les réseaux créent et soutiennent les changements, les moments de mise en service d'une nouvelle action, comme un dispositif, ce qui est assez complexe. Un réseau crée une identité de groupe quand il soude et renforce les prises de décision. Les échanges facilitent la diffusion des apprentissages et les enseignants et professionnels peuvent apprendre en s'enrichissant de l'expérience de l'autre. Un réseau peut diffuser les solutions des problèmes et les analyses des besoins des professionnels. Penser à la FBPF, c'est comprendre que si nous sommes là, c'est parce que le travail collaboratif et bénévole existe depuis longtemps, d'autres l'avaient déjà entamé avant nous.

Qui participe au réseau fédératif brésilien ? Cet effet en cascade stimule les enseignants, qui vont dans des associations, celles qui adhérent à une fédération. Les membres des associations au 


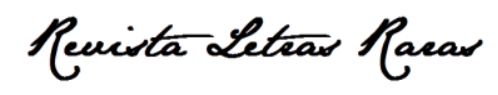

ISSN: 2317-2347 - v. 9, Dossier spécial (2020)

Todo o conteúdo da RLR está licenciado sob Creative Commons Atribuição 4.0 Internacional

Brésil sont généralement des enseignants de français langue étrangère, des étudiants en licence de Lettres ou de Traduction, ainsi que des professionnels liés à la promotion du français au Brésil dans le but de promouvoir la langue française à partir des liens nationaux et internationaux. II y a également plusieurs professionnels et futurs professionnels ainsi que des francophiles, ceux qui aiment la langue française sous toutes ses formes.

La FBPF peut rassembler des associations de tous les états du Brésil, mais actuellement, il y a quatre états sans aucun mouvement associatif3. Penser à l'histoire de la FBPF, c'est évoquer l'histoire des APF, comme par exemple, celle de l'Etat de Minas Gerais, fondée en 1954, celle de l'Etat de São Paulo qui avait été créée en 1961. En 1963, naît l'Association des Professeurs de Rio de Janeiro. En 1968, l'Association des Professeurs de français du District Fédéral, en 1970, de l'Etat de Bahia et un an plus tard, l'Association des Professeurs de français de l'Etat du Pará. A partir de I'histoire des Associations des professeurs des états et du DF (District Fédéral), la FBPF a découvert la potentialité de la vie associative, transformée en vie fédérative, grâce au projet fédérateur qui s'annonçait dans les années 1970/1980.

Ces associations rassemblées visaient plus de visibilité dans le contexte national la Fédération elle-même a une histoire, un contexte. C'est comme cela que le 1er août 1975, à l'Université de Brasilia (UnB), institution appelée l'Association brésilienne des professeurs universitaires de français a vu le jour. Des réunions ont eu lieu dans les années 1976 et 1977 avec un congrès scientifique à Rio de Janeiro. Ensuite, d'autres réunions en 1978, 1979, 1980 et un congrès national à Fortaleza en 1983. En 1985, s'est tenue une réunion des représentants des États et en 1987, un congrès au Rio Grande do Sul, suivi d'un autre en 1988, à l'Etat d'Alagoas, puis en 1991 dans l'Etat de Santa Catarina, à Florianópolis. Deux années plus tard, un congrès à João Pessoa (Paraíba) en 1993, quand cette association choisit l'appellation Fédération brésilienne des Professeurs de Français.

Pour pérenniser les actions menées et les décisions prises, nous passons à l'écrit. Ensuite, il faut valider les procès-verbaux (PV) auprès des instances juridiques responsables. Et c'est justement à travers la lecture des procès-verbaux de la fédération que nous comprenons la potentialité de la vie

\footnotetext{
${ }^{3}$ Selon le site de l'entité (www.fbpf.org.br), il n'y a aucune représentation auprès de la FBPF dans les Etats de Rondônia, Tocantins, Amazonas et Maranhão. Cela ne veut pas dire que la langue française n'existe pas dans ces états où qu'il n'y avait jamais eu d'associations dans ces états. Cela veut dire qu'en 2020, personne n'a décidé de représenter la vie associative auprès de la FBPF.
} 


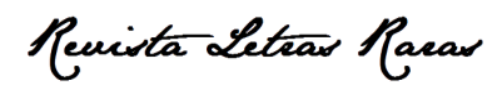

ISSN: 2317-2347 - v. 9, Dossier spécial (2020)

Todo o conteúdo da RLR está licenciado sob Creative Commons Atribuição 4.0 Internacional

associative. Ces PV nous montrent que le travail collaboratif réalisé au sein d'une fédération dévoile l'importance des actions pour promouvoir la formation enseignante, soit à partir du partenariat avec d'autres agents qui travaillent pour la diffusion de la langue française, soit avec des groupes de recherches, des membres des bureaux des associations locales qui s'engagent pour diffuser les cultures d'expression française. Les PV révèlent des rapports narratifs et financiers des conventions de subventions signées, l'organisation des événements scientifiques nationaux et les projets pour le renforcement du FLE dans les programmes des systèmes éducatifs publics et privés au Brésil.

\subsection{Les défis des associations solides, des associations débutantes et « dormantes »}

Le site de la FBPF indique les états où il y a une vie associative au Brésil. Ce site est devenu un espace d'échange et de partage (CHARLIER, 2010). Selon Charlier (2010), le mot échange sera compris comme communication et le mot partage, comme " prendre part ». II faut souligner que la catégorie « participation » est fondamentale dans la théorie des communautés de pratiques ${ }^{4}$.

A partir de cette rubrique, nous vérifions qu'il y a plusieurs profils associatifs. II y a des associations solides qui s'organisent juridiquement et du côté administratif et fiscal. II y a des associations débutantes, celles qui (re)commencent leurs activités, qui préparent des bureaux provisoires et qui essaient de rassembler d'autres professionnels pour cette action de collaboration. Nous constatons également des états au Brésil où il n'y a qu'un représentant local, quelqu'un qui répond auprès de la FBPF pour des possibles mouvements associatifs dans leur région : ce sont des états où la vie associative est si dormante qu'il n'y a aucun mouvement détecté.

Les défis des associations solides dans le pays et des associations débutantes, y compris celles qui recommencent leurs activités s'inscrivent dans le domaine de la gestion. Gérer une association, c'est un choix et une décision pris par des professionnels qui partiront de façon bénévole auprès des instances juridiques et fiscales pour faire valider leurs PV, ayant comme aide professionnelle les bureaux de comptabilité, ceux qui guident les dirigeants dans les obligations auprès du représentant des impôts : les bureaux de la Receita Federal au Brésil. Alors, plusieurs actions de gestions sont requises : administratives, juridiques, fiscales. C'est un choix que de mettre son temps

\footnotetext{
${ }^{4}$ Cet article ne prétend pas réfléchir de façon approfondie sur la thématique concernant les communautés de pratiques.
} 


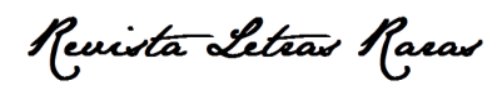

ISSN: 2317-2347 - v. 9, Dossier spécial (2020)

Todo o conteúdo da RLR está licenciado sob Creative Commons Atribuição 4.0 Internacional

au service collectif et aussi de prévoir le montant nécessaire pour les frais juridique, fiscal et administratif. A part ce côté objectif, il y a une question plutôt subjective : gérer les critiques des pairs et abandons des membres. Pour cela, il faut toujours comprendre le moment sociohistorique et le contexte professionnel auxquels le collectif d'enseignant est soumis.

Les défis pour ceux et celles qui décident de relancer les associations "dormantes » retombent sur ce que nous appellerons l'effet : "Mon association". Des anciens présidents et présidentes ainsi que des membres de bureaux précédents décident que l'association est un bien privé et gardent les PV et les documents dans un placard fermé à clé. Sans pouvoir retrouver le bon chemin de l'association qui avait déjà existé, le nouveau groupe de professionnel désirant faire renaître la vie associative se retrouve empêché. Ce manque de respect auprès de ce qui est public et collectif est une entrave à la reprise de la vie associative active dans le pays. Le meilleur chemin pour cette reprise est de prendre des décisions dans un groupe ayant des anciens membres associés et des membres nouveaux: du passé à la contemporanéité, voici la force du collectif qui décide de revivre une association. A partir de cette décision, il faut se renseigner juridiquement pour comprendre comment le faire, comment agir sans perte de temps. II y a des démarches à suivre et c'est le notaire et le comptable qui auront la parole au premier moment. Tout cela nos démontre qu'une association n'est pas une école, ni un groupe de recherches ou un groupe d'amis. Nous ne sommes pas dans une entreprise, ni dans une institution commerciale. Ce n'est pas un projet d'activités d'extension universitaire. Ce n'est pas un objet à acquérir ni une plateforme liée à un réseau social. Une fédération est, à la limite, une association. Ce qui change, c'est la nature de ses membres. Une association de professeurs a comme adhérents des enseignants et des professionnels et une fédération a des associations.

\subsection{La formation en continue à partir de la vie fédérative, voire associative}

Promouvoir des actions pour la formation didactique des formateurs en français fut le rôle majeur de la FBPF. A partir de la lecture de ses PV, nous relevons les débats liés à la question des méthodes d'apprentissage, des choix pour que des enseignants partent dans le cadre des stages de formationà l'étranger, et aussi tout l'effort pour organiser des événements scientifiques prouvent cette 


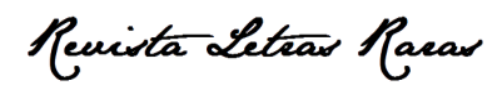

ISSN: 2317-2347 - v. 9, Dossier spécial (2020)

Todo o conteúdo da RLR está licenciado sob Creative Commons Atribuição 4.0 Internacional

préoccupation commune. Penser aux formations continues veut dire au préalable évaluer les contextes et les besoins des enseignants.

Une question s'impose : qui enseigne le français au Brésil ? Nous avons plusieurs contextes professionnels. Celui les enseignants qui travaillent dans des universités; Oui, ok! D'autres enseignants sont indépendants et enseignent chez eux comme des enseignants autonomes donnant des cours privés et sur l'Internet. Pendant la crise sanitaire de 2020, nous avons vu surgir plusieurs enseignants autonomes sur les réseau sociaux et plateformes de media. En 2020 également, nous avons vu la reprise de la formation Professionnalisation en Français Langue Étrangère (Profle) au Brésil, une formation non diplômante comme cours à distance en ligne. Nous avons observé également que les enseignants ont pu suivre des webinaires, des conférences, des débats en ligne en 2020 et ils ont aussi pu élargir leur rôle d'enseignants à partir d'autres activités, comme celles de tutorat et d'animation de plateforme type IFprof Brésili5. De quoi ont-ils besoin pour se former ? Si en 2020 la mise en service de dispositifs de formation à distance a été nécessaire, dans les années précédentes la FBPF, en partenariat avec l'Ambassade de France et avec la Fédération internationale des professeurs de français, a offert des formations en présentiel.

\subsubsection{Penser à une formation continue au-delà des frontières de l'enseignement du français}

L'année 2020 est assez particulière à cause de la pandémie du nouveau Coronavirus. Nous avons constaté que la FBPF s'est rapprochée d'autres associations congénères. Une activité menée pour la formation continue proposée par le bureau de la FBPB en 2020 fut la participation à un webinaire le 24 juin sur la chaîne YouTube en partenariat avec l'Association de Linguistique Appliquée brésilienne qui a traité des matériaux didactiques en langue étrangère : en anglais, en espagnol et en français. Ce tour d'horizon didactique resserre les avec d'autres pairs qui exercent un travail semblable dans d'autres langues. L'appel à candidature proposé en mars 2020, avant la crise sanitaire, pour suivre un Master en Français Langue Etrangère à l'Université des Antilles à distance renforce l'idée que les partenariats avec le service de coopération éducative de l'Ambassade de France au Brésil

\footnotetext{
5 Selon le site https:/lifprofs.org/br, la plate-forme IFprof est un réseau social de l'éducation en français dont les responsables sont l'Institut Français et Ministère de l'Europe et des Affaires étrangères.
} 


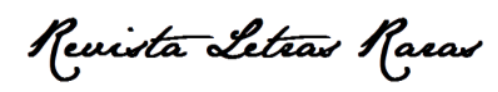

ISSN: 2317-2347 - v. 9, Dossier spécial (2020)

Todo o conteúdo da RLR está licenciado sob Creative Commons Atribuição 4.0 Internacional

valorisent les actions menées pas seulement sur l'hexagone et l'Amérique, mais en distanciel, ce qui a été très bien apprécié durant cette pandémie.

Sur le site de la Fédération, il y a une rubrique nommée: "politiques éducatives et linguistiques ». Cette ouverture vers le contexte spécifique brésilien procure aux enseignants des informations sur la politique éducative générale, sur la politique de l'éducation bilingue dans le pays et aussi des rapports, appels à publications et participation à des recherches liées à l'éducation et à la linguistique. Nous constatons des mouvements de la FBPF dans la discussion sur les Diretrizes Curriculares Nacionais da Educação Bilingue ${ }^{6}$.

\subsubsection{Former les cadres associatifs : des bénévoles, mais avant tout des professionnels}

Un autre problème est observé auprès des bureaux de gestions de la fédération : celui de Former les cadres associatifs. A partir d'une analyse du site de l'entité comme espace de partage de la FBPF, le constat avéré est que ce site devient un lieu pour communiquer et faire pérenniser les projets et actions. A la rubrique Projet, en 2010 , sont visibles les actions menées afin de souder les liens entre les associations des professeurs. A travers la description de ce projet, une preuve des actions collaboratives au sein de la fédération.

II faut souligner aussi que la délégation de l'Ambassade de France au Brésil est un partenaire majeur de la FBPF. Pour analyser des actions plus récentes, nous citons qu'en 2017, il y a eu un appel pour le départ d'environ 15 professionnels pour un stage organisé pour former le cadre associatif7. Nous constatons également l'existence d'une rencontre pour les présidents des associations des professeurs de français qui atteindra sa dixième édition en 2020, peut-être 2021 en raison de à la crise sanitaire.

Afin rassembler les membres des bureaux des associations des professeurs de français en 2020, dû à la crise sanitaire, la FBPF a décidé d'organiser des rencontres virtuelles entre ses membres.

\footnotetext{
${ }^{6}$ Ce document traite des Référentiels nationaux pour l'éducation bilingue dans le pays. La FBPF a participé à cette discussion à travers un document envoyé en juin 2020. Le document final n'a pas encore été homologué, mais la version finale contient les contributions de la FBPF. Une analyse de contenu pourra se faire dès que le document final sera homologué par le Ministère d'Education au Brésil à partir des données disponibles sur : http://fbpf.org.br/pel_bresil_informations.htm

7 Voir la rubrique Appel /Stages/ France : Lettre du 23 novembre 2017 avec le résultat de cet appel sur le site de l'entité disponible sur le site <http://www.fbpf.org.br>.
} 


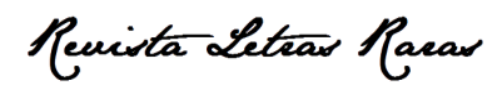

ISSN: 2317-2347 - v. 9, Dossier spécial (2020)

Todo o conteúdo da RLR está licenciado sob Creative Commons Atribuição 4.0 Internacional

Cette initiative a été est motivée suite à la demande d'une présidente d'association lors de l'Assemblée Générale de la FBPF.8 Cette action s'est intitulée " Parole aux APF : une action interne, dont le but a été de rapprocher les membres associés ${ }^{9}$. Écouter les actions de ses paires peut nous former. Les rencontres ont eu lieu sur la plateforme virtuelle Skype et d'une durée d'une heure trente. Dans chaque rencontre, nous avons eu entre trois et quatre associations qui prenaient la parole pour présenter leur contexte et leurs membres des bureaux. Au total 23 associations se sont présentées le 3 et 10 juillet, le 07 et 14 août et le 11 et 18 septembre. L'intérêt de ces rencontres a été rompre l'isolement des APF au Brésil et l'échange des actions en 2020.

\subsubsection{L'insertion professionnelle au cœur de la formation continue}

La thématique liée à l'insertion professionnelle est très actuelle et plusieurs chercheurs se lancent dans des études et recherches, soit dans le contexte de l'enseignant de l'éducation de base, soit dans le contexte des enseignants débutants universitaires. Récemment, un dossier spécial10 sur cette thématique a été lancé par des chercheurs brésiliens dans le but de mieux comprendre les enjeux de la carrière enseignante. Selon André, Passos et Almeida (2020) qui ont coordonné ce dossier, la production scientifique sur cette thématique nous montre que l'insertion professionnelle est une période qui ne peut pas être invisible par les politiques publiques et qu'il existe un éventail de nouvelles situations qui se sont imposées dans la réalité scolaire qui exigent d'autres «apprentissages des enseignants dans leur contexte professionnel et la quête de possibilités et procédures, pas forcément connus et expérimentés auparavant » (ANDRÉ ; PASSOS ; ALMEIDA, 2020, p. 02)

A part la formation didactique des enseignants de français et afin de faire avancer la qualité de cet enseignement et aussi la formation pour le cadre associatif, nous remarquons une avancée vers des actions pour l'insertion professionnelle des enseignants de français. Cette analyse ${ }^{11}$ de quelques rubriques du site de la FBPF nous montre que la fédération elle-même et aussi ses membres, les associations des professeurs de français dans le pays ont mené des actions pour l'insertion

\footnotetext{
${ }^{8}$ Voir le PV de l'Assemblée Générale sur le site de la FBPB disponible sur le site <http://www.fbpf.org.br>.

9 Voir la rubrique Projet / 2020/ Paroles aux Associations disponible sur le site <http://www.fbpf.org.br>.

10 Voir http://www.reveduc.ufscar.br/index.php/reveduc/issue/view/38/showToc

11 Cette analyse n'a pas été exhaustive. Il y a un champ ouvert à des recherches sur la vie fédérative au Brésil à partir de cet espace de partage.
} 


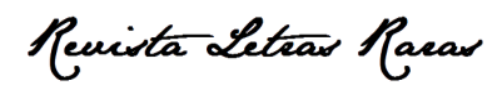

ISSN: 2317-2347 - v. 9, Dossier spécial (2020)

Todo o conteúdo da RLR está licenciado sob Creative Commons Atribuição 4.0 Internacional

professionnelle enseignante. Les professeurs débutants ont eu l'occasion de partir en stage pédagogique organisé en $2018^{12}$. Étant donné la crise sanitaire et les difficultés liées à la précarité de postes, les mouvements pour l'insertion professionnelle sont toujours les bienvenus.

Le choix pour former les enseignants débutants trouve écho dans l'œuvre de Mukamurera (2014 ; 2015). Parmi les dimensions étudiées par cette chercheure sur le développement professionnel des enseignants, nous avons

[...] une dimension collective relative aux habilités collaboratives devenues nécessaires à une à une époque où la collégialité, le partage d'expertise et la collaboration entre les acteurs (enseignants, personnels non enseignants, partenaires de la communauté) sont nécessaires pour la réussite scolaire et institutionnelle [...] (MUKAMURERA, 2014, s/p).

Selon cette auteure, le développement professionnel de l'enseignant est constitué de trois étapes dont la première serait la phase de formation initiale, la seconde, la phase d'insertion professionnelle et la troisième la phase de la formation continue dommage qu'il y ait autant de reprise de mots. Elle constate que si ces phases sont vécues séparément, sans une concertation au préalable, il est possible de «limiter le potentiel de développement professionnel et les retombées perçues » (MUKAMURERA, 2014, s/p).

Mukamurera (2015) nous apprend que le processus d'insertion professionnelle est temporel, dynamique et multidimensionnel, pour cela, il faut soutenir les enseignants débutants, en plus, au début, il faut aussi leur apporter un soutien émotionnel. Les deux premières années sont fondamentales selon l'auteure. II faut penser à une gradation du soutien dans le temps et une prise en compte des besoins réels des enseignants et des situations particulières de leur travail. Les novices demandent un temps commun pour la planification des activités. Ils ont besoin de leurs pairs.

Nieuwenhoven et Mosnenck (2014) affirment comme résultats de leurs recherches et études que les enseignants débutants veulent « partager leurs pratiques, d'échanger entre eux, de favoriser la collaboration avec leurs pairs tant débutants qu'expérimentés » (NIEUWENHOVEN ; MOSNENCK, 2014, s/p). Selon ces auteures, « Ce souhait s'inscrit dans un but de réassurance et de renforcement de l'estime de soi, mais aussi dans un projet de collaboration plus large » (NIEUWENHOVEN ; MOSNENCK, 2014, s /p).

12 Voir la rubrique Appel / Appel de la FBPF/2018 : Appel n. 01/2018 sur le site <http://www.fbpf.org.br>. 


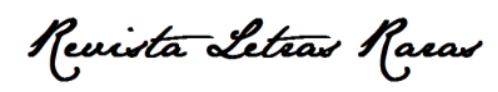

ISSN: 2317-2347 - v. 9, Dossier spécial (2020)

Todo o conteúdo da RLR está licenciado sob Creative Commons Atribuição 4.0 Internacional

Alors, le choix d'envoyer des enseignants novices pour suivre un stage a été pris par la FBPF dont le but de les accompagner dans l'entrée professionnelle. A part la question didactique, il y a l'immersion dans un contexte de langue dans un pays francophone, surtout quand le débat sur la la qualité de l'enseignement mené par un natif ou non natif est encore présent.

\section{En guise de conclusion}

Dans le domaine éducationnel et didactique, penser aux groupes et au travail collaboratifs nous permet d'analyser des actions et des projets sous le prisme des catégories liées au développement professionnel de l'enseignant et de l'insertion professionnel des enseignants débutants.

Les associations des enseignants de langues étrangères sont des organisations sociales privées qui rassemblent des enseignants d'une certaine langue étrangère et d'une région du pays. Elles se réunissent pour former une fédération dont a rendu compte cet article. Leurs membres décident de s'organiser de façon bénévole à partir de buts communs afin d'avancer dans leurs carrières enseignantes. Quelques expériences associatives, fédératives et de la coopération éducative d'organismes étrangers en partenariat peuvent renforcer la profession enseignante en FLE dans le pays.

CEuvrer pour que des professionnels aient des objectifs communs, d'échange, de partage pour les faire avancer dans leur profession et carrière enseignante, visant à un bien commun, est un défi pour ceux et celles qui décident de participer à la vie associative, voire fédérative. Les enjeux actuels de la FBPF sont liés à la valorisation de ce travail collaboratif et bénévole dans une société où les intérêts individuels peuvent surmonter ceux de la collectivité. Les fruits de ces actions concertées dont les retombées seront enregistrées dans l'histoire de la Fédération et de l'enseignement du français dans le pays seront jugés et analysés par cette communauté d'enseignants.

Nous comprenons que nous sommes devant un champ ouvert pour de nouvelles recherches sur le développement professionnel de l'enseignant et sur l'insertion professionnelle enseignante. Et la réflexion sur d'autres espaces possibles de formation enseignante, comme celui des associations des enseignants, en parallèle à l'institution scolaire, peut nous fournir d'autres pistes pour comprendre cette réalité si complexe qu'est la profession enseignante. 


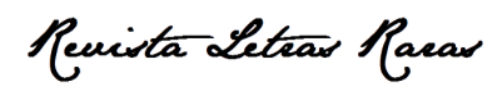

ISSN: 2317-2347 - v. 9, Dossier spécial (2020)

Todo o conteúdo da RLR está licenciado sob Creative Commons Atribuição 4.0 Internacional

\section{Références}

ANDRÉ, M. E. D. A. de; PASSOS, L. F.; ALMEIDA, P. Apresentação. Dossiê "Formação e inserção profissional de professores iniciantes: conceitos e práticas". In: Revista Eletrônica de Educação REVEDUC, v. 14, 1-3, e4780110, jan./dez. 2020. ISSN 1982-7199 | DOI: http://dx.doi.org/10.14244/198271994780. Lien disponible sur http://www.reveduc.ufscar.br/index.php/reveduc/article/view/4780/1075

BOIES, I. ; PORTELANCE, L. La collaboration dans les milieux d'accueil des enseignants débutants. In : MUKAMURERA, J. ; PORTELANCE, L. ; MARTINEAU, S. Développement et Persévérance Professionnels dans l'Enseignement : oui, mais comment? (French Edition - Kindle Edition). Presses Universitaires du Québec, 2014, Kindle Edition.

CALVO, G. Desarrollo profesional docente: el aprendizaje profesional colaborativo. In: Temas críticos para formular nuevas políticas docentes en América Latina y el Caribe: El debate actual. - Santiago, Chile: Organización de las Naciones Unidas para la Educación, la Ciencia y la Cultura. Oficina Regional de Educación para América Latina y el Caribe (OREALC/UNESCO Santiago), 2014, p. 112153.

CHARLIER, B. L'échange et le partage de pratiques d'enseignement au cœur du développement professionnel ? Education et formation, e-293, 2010.

FULLAN, M.; Hargreaves, A. A escola como organização aprendente. Rio de Janeiro : Editora Artmed, 2000.

MUKAMURERA, J. Le développement professionnel et la persévérance en enseignement. Éclairage théorique et état des lieux. In: MUKAMURERA, J. ; PORTELANCE, L. ; MARTINEAU, S. Développement et Persévérance Professionnels dans l'Enseignement : oui, mais comment ? (French Edition - Kindle Edition.). Presses Universitaires du Québec, 2014.

NIEUWENHOVEN, C. Van ; MOSNENCK, S. Van. L'identification des besoins d'accompagnement des étudiants du préscolaire en vue d'améliorer leur insertion professionnelle. In : MUKAMURERA, J. ; PORTELANCE, L. ; MARTINEAU, S. Développement et Persévérance Professionnels dans l'Enseignement : oui, mais comment ? (French Edition - Kindle Edition). Presses Universitaires du Québec, 2014.

PASSOS, L. F. Práticas Formativas em Grupos Colaborativos: das ações compartilhadas à construção de novas profissionaliades. In: ANDRË, M. E. D. A. de (Orgs). - Campinas, SP: Papirus, 2016 (Prática Pedagógica). 